Louisiana State University

LSU Digital Commons

Faculty Publications

School of Animal Sciences

$12-1-2012$

\title{
Jak/Stat3 signaling promotes somatic cell reprogramming by epigenetic regulation
}

\author{
Yong Tang \\ University of Connecticut \\ Yan Luo \\ University of Connecticut \\ Zongliang Jiang \\ University of Connecticut \\ Yinghong $\mathrm{Ma}$ \\ University of Connecticut \\ Chih Jen Lin \\ University of Connecticut
}

See next page for additional authors

Follow this and additional works at: https://digitalcommons.Isu.edu/animalsciences_pubs

\section{Recommended Citation}

Tang, Y., Luo, Y., Jiang, Z., Ma, Y., Lin, C., Kim, C., Carter, M., Amano, T., Park, J., Kish, S., \& Tian, X. (2012). Jak/Stat3 signaling promotes somatic cell reprogramming by epigenetic regulation. Stem Cells, 30 (12), 2645-2656. https://doi.org/10.1002/stem.1225

This Article is brought to you for free and open access by the School of Animal Sciences at LSU Digital Commons. It has been accepted for inclusion in Faculty Publications by an authorized administrator of LSU Digital Commons.

For more information, please contact ir@lsu.edu. 


\section{Authors}

Yong Tang, Yan Luo, Zongliang Jiang, Yinghong Ma, Chih Jen Lin, Chul Kim, Mark G. Carter, Tomokazu Amano, Joonghong Park, Sharon Kish, and Xiuchun Tian 


\title{
Embryonic Stem Cellis/Induced Pluripotent Stem Cells
}

\section{Jak/Stat3 Signaling Promotes Somatic Cell Reprogramming by Epigenetic Regulation}

\author{
Yong Tang, Yan Luo, Zongliang Jiang, Yinghong Ma, Chih-Jen Lin, Chul Kim, Mark G. Carter, \\ Tomokazu Amano, Joonghong Park, Sharon Kish, Xiuchun (Cindy) Tian \\ Center for Regenerative Biology, Department of Animal Science, University of Connecticut, \\ Storrs, Connecticut, USA
}

Key Words. Cell signaling • Embryonic stem cells • Epigenetics • Induced pluripotent stem cells $\bullet$ Reprogramming

\begin{abstract}
Although leukemia inhibitory factor (LIF) maintains the ground state pluripotency of mouse embryonic stem cells and induced pluripotent stem cells (iPSCs) by activating the Janus kinase/signal transducer and activator of transcription 3 (Jak/Stat3) pathway, the mechanism remained unclear. Stat3 has only been shown to promote complete reprogramming of epiblast and neural stem cells and partially reprogrammed cells (pre-iPSCs). We investigated if and how Jak/Stat3 activation promotes reprogramming of terminally differentiated mouse embryonic fibroblasts (MEFs). We demonstrated that activated Stat3 not only promotes but also is essential for the pluripotency establishment of MEFs during reprogramming. We further demonstrated that during this process, inhibiting Jak/Stat3 activity blocks demethylation of Oct4 and Nanog regulatory elements in induced cells, which are marked by suppressed endogenous pluripotent gene expression. These are
\end{abstract}

correlated with the significant upregulation of DNA methyltransferase (Dnmt) 1 and histone deacetylases (HDACs) expression as well as the increased expression of lysinespecific histone demethylase 2 and methyl CpG binding protein 2. Inhibiting Jak/Stat3 also blocks the expression of Dnmt3L, which is correlated with the failure of retroviral transgene silencing. Furthermore, Dnmt or HDAC inhibitor but not overexpression of Nanog significantly rescues the reprogramming arrested by Jak/Stat3 inhibition or LIF deprivation. Finally, we demonstrated that LIF/ Stat3 signal also represents the prerequisite for complete reprogramming of pre-iPSCs. We conclude that Jak/Stat3 activity plays a fundamental role to promote pluripotency establishment at the epigenetic level, by facilitating DNA demethylation/de novo methylation, and open-chromatin formation during late-stage reprogramming. STEM CELLS 2012;30:2645-2656

Disclosure of potential conflicts of interest is found at the end of this article.

\section{INTRODUCTION}

Induced pluripotent stem cells (iPSCs) are embryonic stem cell (ESC)-like cells reprogrammed from differentiated cells by overexpression of reprogramming factors including Oct4 (also known as Pou5f1), Klf4, Sox2, and c-Myc (OKSM) or Oct4, Sox2, Nanog, and Lin28 [1-3]. The iPSC technology holds great potential for regenerative medicine in producing patient-specific pluripotent cells while bypassing embryo manipulations. Methodologically, the induction of iPSCs has evolved rapidly, from the initial viral transduction to recombinant proteins, modified mRNAs, or microRNAs [4-8]. However, the underlying mechanism for the molecular reprogramming remains ill-defined.

Activation of gene expression involves complicated epigenetic events such as DNA demethylation and histone methylation/acetylation, which render an open-chromatin structure for the full access of transcriptional complexes [9-11]. Complete reprogramming of somatic cells is marked by the full activation of the Oct4 gene-the master regulator of pluripotency [12-14]. Accordingly, the Oct4 and Nanog enhancer/promoter elements are hypermethylated in somatic cells and hypomethylated in ESCs $[2,15,16]$, whereas the histone hyperacetylation is closely associated with Oct4 and Nanog regulatory regions in ESCs, with hypoacetylation of these regions in somatic cells [2, 15].

Silencing of retroviral transgenes is a prerequisite for pluripotency establishment in retroviral transgene-mediated reprogramming, as overexpression of viral transgenes, especially Oct4, causes differentiation of pluripotent cells $[13,17$, 18]. Retroviral silencing is achieved by de novo DNA methylation through DNA methyltransferases (Dnmts) 3a, 3b, and Dnmt3L. Although Dnmt3L lacks a functional catalytic domain, it directly interacts with Dnmt3a/3b and significantly enhances their activities [19-22]. Knocking-down of Dnmt3L renders the ESCs incapable of silencing newly incorporated retroviral DNA, demonstrating the essential role of Dnmt3L expression in retroviral silencing [23].

Author contributions: Y.T.: conception and design, provision of study material, collection and/or assembly of data, data analysis and interpretation, and manuscript writing; Y.L., Z.J., Y.M., and C-J.L.: provision of study material and collection and analysis of data; C.K., M.C., T.A., J.P., M.A., and S.K.: provision of study material; X.C.T.: conception and design, data analysis and interpretation, financial and administrative support, and manuscript writing.

Correspondence: Xiuchun (Cindy) Tian, Ph.D., Center for Regenerative Biology, Department of Animal Science, University of Connecticut, 1390 Storrs Road, Storrs, Connecticut 06269, USA. Telephone: 860-486-9087; Fax: 860-486-3536; e-mail: xiuchun.tian@ uconn.edu Received May 9, 2012; accepted for publication August 14, 2012; first published online in STEM CelLS EXPRESS September 11, 2012. (C) AlphaMed Press 1066-5099/2012/\$30.00/0 doi: 10.1002/stem.1225

Stem Cells 2012;30:2645-2656 www.StemCells.com 
Activation of Janus kinase/signal transducer and activator of transcription 3 (Jak/Stat3) signal pathway maintains mouse ESC pluripotency [24]. Jak/Stat3 signal pathway is mainly activated by leukemia inhibitory factor (LIF), which also activates the phosphatidylinositol 3-kinase (PI3K)/protein kinase $\mathrm{B}$ (also known as Akt), and the extracellular-signal-regulated kinases 1/2 (Erk1/2) pathways in ESCs [25-27]. LIF activates these pathways through ligand-induced heterodimerization between LIF receptor and the signal transducer protein gp130 $[28,29]$. Artificially activated Stat 3 sustains ESC self-renewal in the absence of LIF [30]. Little is known about the downstream mechanism of Stat3 in pluripotency maintenance, although it has been shown that LIF/Stat3 upregulates Klf4 and Myc expression in mouse ESCs [25, 31, 32], and Stat3 has been indicated to antagonize the differentiation signal of Erk1/2 [33, 34].

The enhanced Stat 3 activity has been shown to promote complete reprogramming from mouse epiblast stem cells, neural stem cells, and OKSM-induced pre-iPSCs, which form colonies but do not express key pluripotent genes such as Oct4 and Nanog [35]. However, the underlying mechanism by Stat3 during reprogramming remained unclear. In addition, it was unknown whether LIF/Stat3 signaling can promote direct reprogramming from terminally differentiated somatic cells. Using mouse embryonic fibroblasts (MEFs) harboring the green fluorescent protein (GFP) transgene driven by the Oct4 gene regulatory region (Oct-GFP-MEFs), we investigated these questions and presented evidence to demonstrate that the enhanced Stat 3 activity improves direct MEF reprogramming, and that Jak/Stat3 activity is essential for reprogrammed cells to attain pluripotency. The underlying mechanism involves stimulation of DNA demethylation and de novo methylation through regulating the expressions of Dnmts as well as suppressing the expression of the major repressivechromatin relevant genes. We also show that inhibiting Dnmt1 and histone deacetylases (HDACs) resumes reprogramming in the presence of Jak/Stat3 inactivation. We propose a model of Jak/Stat 3 signaling to promote murine somatic cell reprogramming where Jak/Stat3 plays a primary role for pluripotency accomplishment at late-stage reprogramming through epigenetic regulations.

\section{RESUlTS}

\section{Enhanced Stat3 Activation Promotes Direct MEF Reprogramming}

We used a constitutively active Stat 3 (Stat3C) with A662C and N664C substitutions in the SH2 domain, which prompts Stat3 dimerization and continued activation [36], and transfected MEFs using Stat3C and the vector control to evaluate its function. As expected, 20 minutes of LIF addition stimulated rapid phosphorylation of Akt, Stat3, and Erk1/2 in MEFs, iPSCs, and ESCs as shown by Western blotting (Fig. 1A). In both the absence and presence of LIF, MEFs transfected with Stat3C exhibited increased levels of phosphorylated Stat 3 compared to the vector control (Fig. 1A). This effect is reminiscent of Stat3ER, a tamoxifen-inducible Stat 3 construct [30]. Quantitative real time reverse transcriptase polymerase chain reaction (qRT-PCR) analysis revealed that Stat3C expression in MEFs significantly stimulated the expression of Socs 3 - the direct Stat 3 target, whose expression is further elevated by additional LIF stimulation (Fig. 1B).

To evaluate whether enhanced Stat 3 activation promotes the reprogramming of terminally differentiated cells, we trans- duced Oct4-GFP-MEFs with viral OKS or OKSM and further transduced them with Stat3C or the control vector. Completely reprogrammed colonies are defined as those exhibiting strong Oct4-GFP expression (GFP+) 2 or 3 weeks after the initial viral transduction (supporting information Fig. S1). In OKS transduction, the expression of Stat3C increased the number of GFP+ colonies by threefold compared to the vector control without LIF, an effect similar to the vector control with LIF treatment (Fig. 1C). The maximum reprogramming effect was achieved by the combination of LIF and Stat3C, producing a 12 -fold increase in the number of GFP+ colonies over that of the control without LIF (Fig. 1C). The LIF/ Stat3C combination not only improved the total colony formation but also significantly improved the percentage of GFP+ colonies $(79 \%)$ compared to the LIF minus control (43\%) (Fig. 1D; supporting information Table S1), indicating that Stat3 activation improves pluripotency achievement among the induced colonies during late-stage reprogramming, defined in this study as the establishment of pluripotency in intermediate colonies. In OKSM-transduced MEFs, we also observed a modest increase ( $\sim 1.6$-folds) in the number of GFP + colonies by LIF/Stat3C combined treatments (Fig. 1E). Importantly, similar to the OKS transduction, the percentage of GFP+ colonies was significantly increased in LIF/Stat3Ctreated cells from $40 \%$ (control) to $79 \%$ (Fig. 1F). Improvement of both OKS and OKSM MEF reprogramming was also observed using the mutant gp130 construct (gp130Y757F), with a phenylalanine to tyrosine substitution at residual position 757, which blocks the activation of PI3K and Erk, and confers constitutive Jak/Stat3 activation upon LIF stimulation [35, 3740] (Fig. 2A, 2B, dimethyl sulfoxide (DMSO) treatment).

Characterization of GFP + colonies from OKS or OKSM plus Stat3C-transduced MEFs demonstrated that these cells were positive for alkaline phosphatase activity, readily grew upon pick-up in 2i/LIF medium - the ground state selective culture medium for ESCs [33, 41], and maintained bright GFP expression and ESC-like colony morphology under repeated passaging (Fig. 1G; supporting information Fig. S2). Testing by qRT-PCR and immunostaining revealed that these cells expressed endogenous ESC-specific genes/markers such as Oct4, Nanog, Sox2, and SSEA-1 (Fig. 1H; supporting information Figs. S3-S6). The induced iPSCs also demonstrated pluripotency in vitro by embryoid body formation with the expression of three germ layer markers (supporting information Figs. S7, S8) as well as the formation of beating cardiac cells upon differentiation in serum-containing medium without LIF (supporting information online video 1). The retroviral Stat3C and Oct4 expressions were also silenced in these iPSCs (supporting information Fig. S9). Taken together, our results demonstrated that the enhanced Stat 3 activity promotes pluripotency establishment during late-stage OKS or OKSM reprogramming of MEFs. In addition, Stat 3 activity also promotes the total colony formation in OKS induced MEF reprogramming.

\section{Jak/Stat3 Activity Is Essential for Pluripotency Establishment in Late-Stage MEF Reprogramming}

It was indicated that the Stat 3 activity represents a rate limiting step in reprogramming, however, whether it is required for complete reprogramming remained unknown [35]. In addition, blocking Jak/Stat3 signaling was shown to improve the neural progenitor cell generation for OKSM transduction of MEFs in a neuronal medium [42]. To address whether Jak/Stat3 activity is required for the complete reprogramming of somatic cells, we reprogrammed Oct4-GFP-MEFs in the presence of LIF and cultured them with either DMSO control or a low dosage $(1 \mu \mathrm{M})$ of the reversible Jak inhibitor I (Jaki) [25, 42], starting 

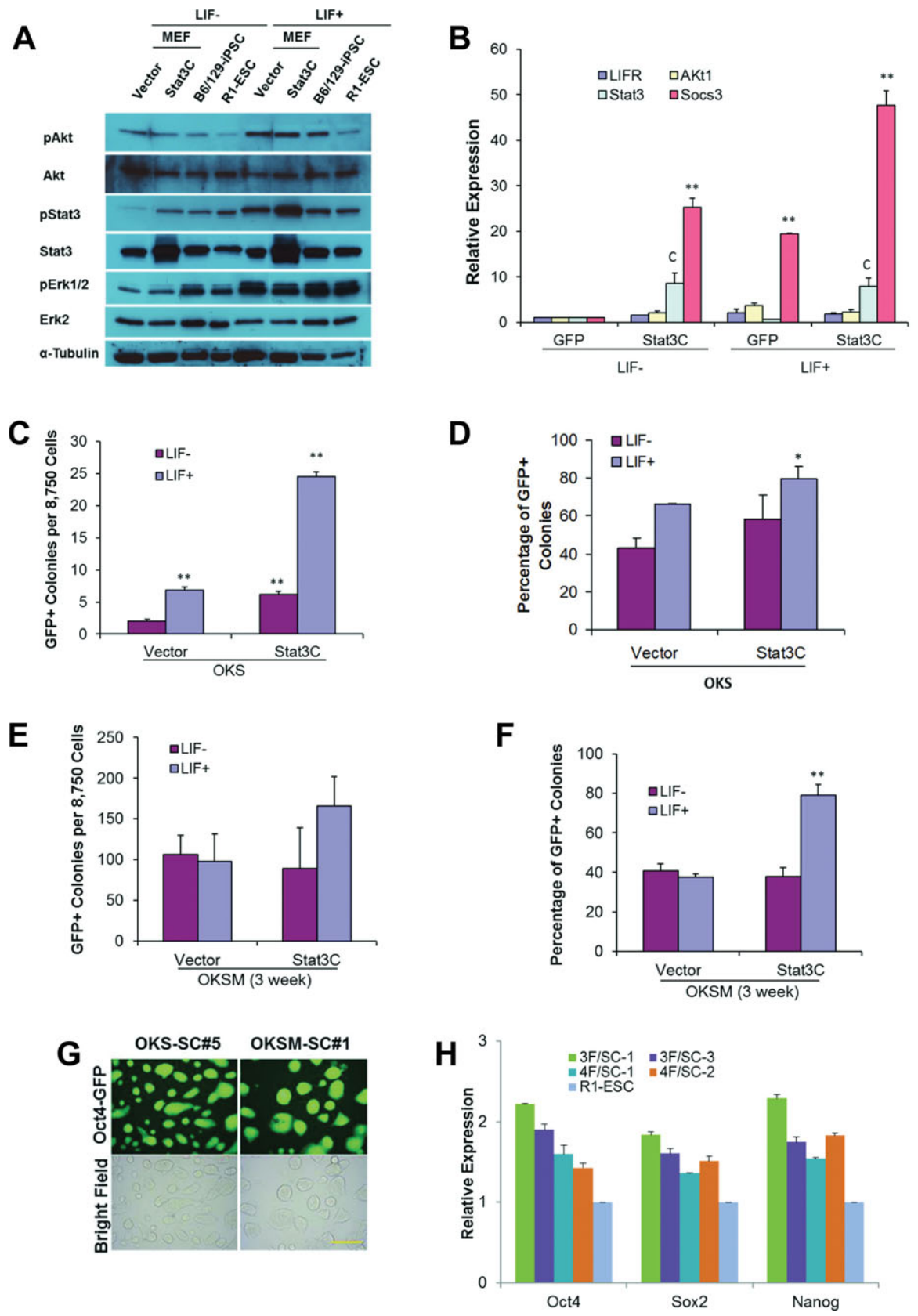

Figure 1. Enhanced Stat3 activation promotes MEFs reprogramming. (A): Western blots of total proteins extracted from B6/129-MEFs, R1ESCs, and B6/129 iPSCs previously generated in our lab using retroviral OKSM. The specific proteins probed were Akt, Stat3, Erk1/2 and their phosphorylated forms. Tubulin was used as the loading control. (B): Relative levels of mRNA (mean \pm SD) for LIF receptor (LIFR), Akt, Stat3, and Socs3 in B6/129 MEFs transfected with retroviral GFP control or Stat3C. "c" indicates the combined endogenous Stat3 and retroviral Stat3C transgene expression. Values were normalized to glyceraldehyde 3-phosphate dehydrogenase (GAPDH) and relative to GFP control cells without LIF stimulation; **, $p=.000, n=2)$. (C, D): The number (mean $\pm \mathrm{SD}$ ) of GFP + colonies (C) and the percentages of GFP + colonies among total ESC-like colonies (D) induced by Stat3C or vector control from Oct4-GFP-MEFs 3 weeks after reprogramming by OKS cultured with or without LIF $(*, p=.0032 ; * *, p=.000 ; n=2)$. $(\mathbf{E}, \mathbf{F})$ : The number (mean $\pm \mathrm{SD}$ ) of GFP + colonies (E) and the percentage (mean $\pm \mathrm{SD})$ of GFP+ colonies among total ESC-like colonies (F) at 3 weeks after retroviral OKSM plus vector or Stat3C transduction of Oct4-GFP-MEFs, cultured in medium with or without LIF (**, $p=.002 ; n=2)$. (G): Representative iPSC colonies at passage 5 induced by OKS plus Stat3C (OKSSC) or OKSM plus Stat3C (OKSM-SC) and cultured in 2i/LIF medium (scale bar $=250 \mu \mathrm{m})$. (H): Relative levels of mRNA (mean \pm SD) for the endogenous Oct4, Sox2, and Nanog in iPSC colonies at passage 6. The values were normalized with GAPDH and relative to R1-ESC (3F: OKS; 4F: OKSM; SC: Stat3C. $n=2$ ). Abbreviations: ESC, embryonic stem cell; GFP, green fluorescent protein; iPSC, induced pluripotent stem cell; LIF, leukemia inhibitory factor; LIFR, LIF receptor; MEF, mouse embryonic fibroblast; OKSM, Oct4, Klf4, Sox2, and c-Myc; Stat3, signal transducer and activator of transcription 3.

at day 3 of viral transduction. Jaki treatment virtually blocked any GFP + colony formation in either OKS- or OKSM-induced MEF reprogramming, regardless of the expression of gp130Y757F (Fig. 2A-2C). However, the formation and the total number of colonies bearing the ESC-like morphology induced by OKSM were not affected by Jaki treatment (Fig. 2C, 2D). In contrast, inhibition of PI3K/Akt activity with 10 $\mu \mathrm{M}$ LY294002 only partially decreased but did not block the 

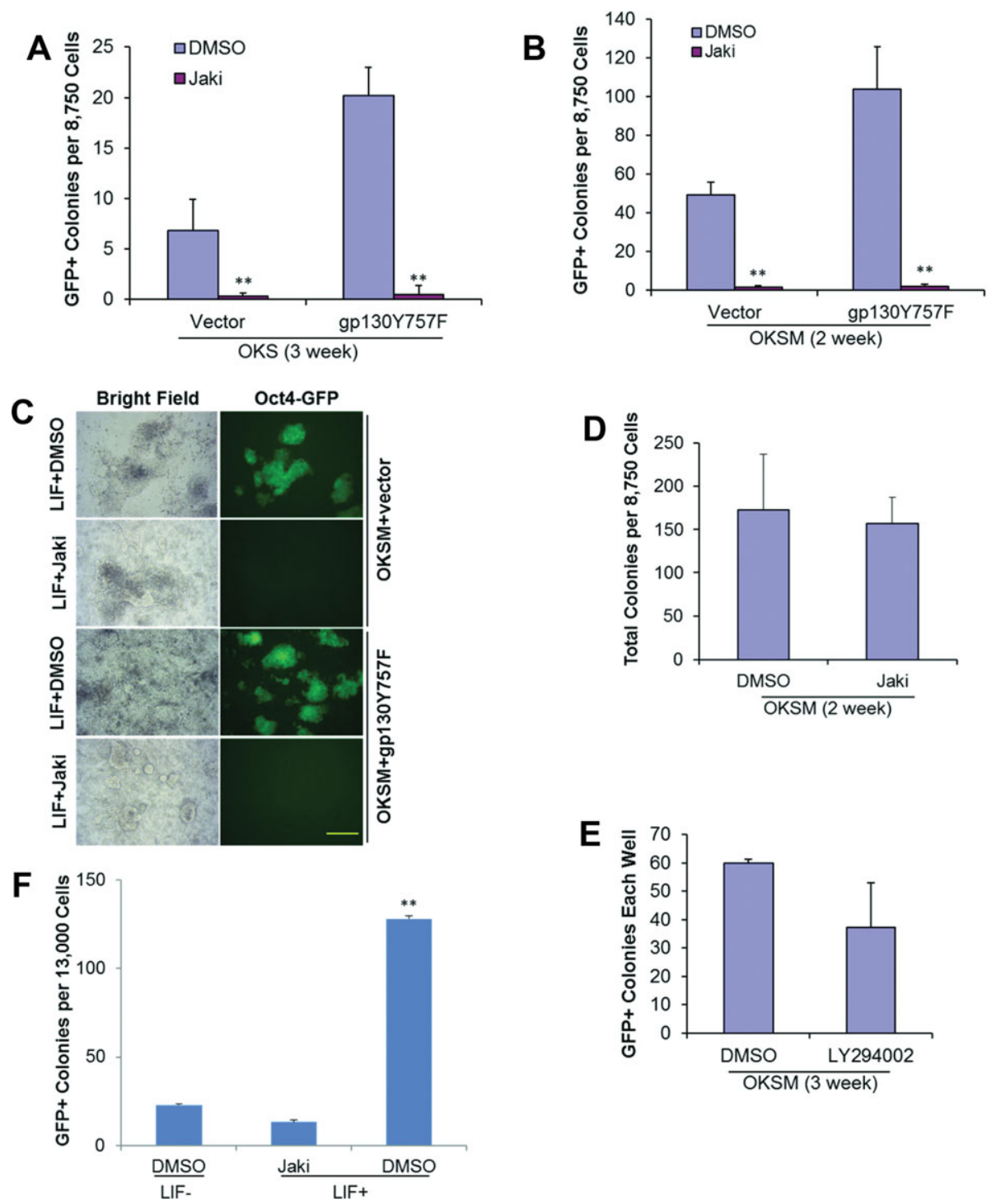

Figure 2. Janus kinase/signal transducer and activator of transcription 3 (Jak/Stat3) activity is essential for complete mouse embryonic fibroblasts (MEFs) reprogramming. (A): The number of GFP + colonies (mean \pm SD) induced by OKS plus gp130Y757F or control retroviral vector, cultured in LIF and in the presence of either DMSO or $1 \mu \mathrm{M}$ Jaki. The colonies were scored at 3 weeks after the initial OKS viral transduction $(* *, p=.000, n=2)$. (B): The number of GFP + colonies (mean $\pm \mathrm{SD}$ ) induced by retroviral OKSM plus gp130Y757F or control vector, cultured with LIF and in the presence of either DMSO or $1 \mu \mathrm{M}$ Jaki. The colonies were scored at 2 weeks after the initial OKSM transduction $(* *, p=.009, n=2)$. (C): Representative embryonic stem cell (ESC)-like colonies induced from Oct4-GFP-MEFs and treated with either DMSO or Jaki as described in $(B)$ (scale bar $=250 \mu \mathrm{m}$ ). (D): The number of total ESC-like colonies (mean \pm SD) induced by OKSM in LIF containing reprogramming medium with DMSO or $1 \mu \mathrm{M}$ Jaki at 2 weeks after viral transduction $(n=2)$. $(\mathbf{E})$ : The number of GFP+ colonies (mean \pm SD) induced by OKSM in LIF and either DMSO or $10 \mu \mathrm{M}$ LY294002. The colonies were scored at 3 weeks after OKSM transduction $(n=2)$. $(\mathbf{F})$ : Number of GFP+ colonies (mean $\pm \mathrm{SD}$ ) induced by OKSM transduction of Oct4-GFP-MEFs reprogrammed without feeder cells, cultured in medium with either DMSO, DMSO + LIF, or $1 \mu \mathrm{M}$ Jaki + LIF 18 days after viral transduction (**, $p=.000, n=2$ ). Abbreviations: DMSO, dimethyl sulfoxide; GFP, green fluorescent protein; LIF, leukemia inhibitory factor; OKSM, Oct4, Klf4, Sox2, and c-Myc; jaki, Jak inhibitor.

formation of GFP + colonies, a result unlikely due to the possible cytotoxicity from LY294002 at this dosage, as judged by the normal morphology and growth characteristics of the reprogrammed cells and colonies under continued treatment (Fig. 2E and data not shown). These results indicate that Jak/ Stat 3 activation not only promotes but also is required for the pluripotency establishment during late-stage reprogramming.

If Jak/Stat3 inhibition blocks pluripotency establishment during reprogramming, then eliminating LIF, the Jak/Stat3activating cytokine, should produce a similar effect. To verify this, we reprogrammed MEFs by retroviral OKSM in the absence of LIF and without feeder cells to minimize LIF in the culture medium [43, 44]. These treatments resulted in a drastic reduction in GFP + colony formation which was nearly identical to inhibiting Jak/Stat3 in the presence of LIF (Fig. 2F). Taken together, these data demonstrated that the LIF-dependent activation of the Jak/Stat3, but not the PI3K/ Akt activity, is essential for the induced colonies to acquire pluripotency during late-stage MEF cell reprogramming.

\section{Jak/Stat3 Activity Promotes Retroviral Silencing and Regulates Dnmt Expression}

To determine how Jak/Stat3 activity regulates the pluripotency establishment during reprogramming, we analyzed the 
mRNA transcripts 18 days after OKSM transduction of MEFs, in the presence of either DMSO or Jaki. While the endogenous pluripotent genes including Oct4, Nanog, and Rex1 are significantly upregulated in reprogrammed cells treated with DMSO control, the expression of these genes in Jakitreated cells, although upregulated from un-reprogrammed MEFs, was at least 7-, 4-, or 70-fold less than those in the DMSO control cells, respectively (Fig. 3A). These results confirmed that Jak/Stat 3 inhibition renders the cells to cease reprogramming at an early-stage of the process. The endogenous Sox 2 mRNA, however, was similarly upregulated in both the DMSO control and Jaki-treated cells (Fig. 3A) compared to the MEF control.

Interestingly, we found that the expressions of all four retroviral transgenes were much greater (3-6-fold) in Jakitreated cells than in the DMSO-treated controls (Fig. 3A). This suggested an impeded de novo DNA methylation to silence the retroviral transgenes [19]. We therefore examined the expression of Dnmts in reprogrammed cells. Testing by qRT-PCR revealed that the expression of de novo methyltransferase Dnmt3a was further upregulated, and the expression of Dnmts $3 \mathrm{~b}$ and $3 \mathrm{~L}$ was stimulated dramatically in DMSO control reprogrammed cells, compared to the parental MEFs (Fig. 3B), indicating increased de novo methylation activities. However, Jak/Stat3 inhibition completely blocked Dnmt3L expression during reprogramming (Fig. 3B). In addition, Jak/Stat3 inhibition resulted in significantly elevated Dnmt1 expression compared to the DMSO control (Fig. 3B). To minimize the heterogeneity of cells in the analysis, we isolated/expanded three typical colonies from each treatment at 3-week after viral transduction and analyzed their gene expression at passage 2. A twofold elevation of Dnmt1 and a complete loss of Dnmt3L expression were consistently observed in Jaki-treated cells, compared to the DMSO controls (Fig. 3C). In addition, Jaki-treated colonies exhibited lower levels of Dnmt3a/3b compared to the DMSO-treated colonies, which showed elevated levels of de novo Dnmts above the ESCs, indicating a further stimulation of de novo methylation activity in these control cells (Fig. 3C). In accordance with these findings, qRT-PCR analysis revealed that while the retroviral transgenes were basically silenced in colonies treated with DMSO control, they remained highly expressed in Jaki-treated cells (Fig. 3D).

The lysine methyltransferase G9a has been shown to recruit Dnmt3a to the endogenous retroviral DNA and is required to establish the silencing of exogenous Moloney murine leukemia viral DNA in ESCs by de novo methylation $[45,46]$. We therefore evaluated the G9a expression in reprogrammed cells and found that its expression was not affected by Jak/Stat3 inhibition (supporting information Fig. S10). Thus, our results demonstrated that Jak/Stat3 stimulates the silencing of retroviral transgenes through activating the expression of Dnmt3L, the essential role of which for retroviral silencing has been demonstrated previously [23].

\section{Jak/Stat3 Activity Promotes DNA Demethylation of Pluripotent Genes and Suppresses the Expression of Repressive Chromatin Relevant Genes during Reprogramming}

As Dnmt1 is responsible for maintaining the existing DNA methylation pattern during cell division [19, 47], and enhanced Dnmt1 expression is directly associated with the accelerated entry into the S-phase as well as cellular transformation [4850], it is therefore not surprising for us to see a greater Dnmt1 expression in the reprogrammed cells and ESCs, which undergo much rapid cell division compared to MEFs (Fig. 3B, 3C). How- ever, we also found that Jak/Stat3 inhibition leads to significantly elevated Dnmt1 expression in reprogrammed cells compared to the control cells (Fig. 3B, 3C). This is correlated with the arrested reprogramming by Jaki treatment, with only low levels of activated endogenous pluripotent genes including Oct4 and Nanog (Fig. 3A, 3D). We then investigated if Jak/Stat3 inhibition directly affects DNA methylation of these genes during reprogramming. DNA pyrosequencing revealed that obvious DNA demethylation happened to the proximal promoter and distal enhancer regions of Oct4 in DMSO-treated control cells, compared to the parental MEFs at day 18 after retroviral transduction (Fig. 3E). However, in cells treated with Jaki, these regions remained hypermethylated, similar to those in MEFs (Fig. 3E). The DNA hypermethylation of the Oct4 and Nanog enhancer/promoter regions in Jaki-treated cells was further verified by bisulfite DNA sequencing (Fig. 3F; supporting information Fig. S11). Thus Jak/Stat3 inhibition blocks the DNA demethylation of pluripotent genes during reprogramming.

Dnmt1 had been identified as a direct target for Stat3, which can either activate or repress the expression of its targets [51]. We evaluated the effect of activated Stat3 on Dnmt1 expression in MEF cells. Four days after retroviral Stat3C transduction, the levels of Dnmt1 mRNA were significantly reduced compared to the control MEFs (Fig. 4A). These results indicate that the Stat 3 activity directly inhibits Dnmt 1 expression and therefore promotes a passive DNA demethylation in reprogrammed cells which are undergoing rapid cell division.

By converting the 5-methylcytosine to the 5-hydroxymethylcytosine $(5 \mathrm{hmC})$ followed by the base excision repair pathway, tet methylcytosine dioxygenase (Tet) proteins were shown to promote active DNA demethylation in mammalian cells [52, 53]. However, Tet 1 also functions to suppress Polycomb-targeted differentiation genes in ESCs $[54,55]$. Tet1/2 expression level increased in induced iPSCs compared to the parental MEFs [56]. We found that during the reprogramming, Tet1 expression increased moderately at day 18 of retroviral iPSC induction, and that Jaki treatment partially inhibited the Tet1 increase in reprogrammed cells (supporting information Fig. 12). However, the small difference in Tet1 expression between DMSO- and Jaki-treated cells at this reprogramming stage does not correlate well with the completely blocked pluripotency establishment by Jaki treatment at 2 -week time point after iPSC induction (Fig. 2B, 2C). Our finding thus does not support an active DNA demethylation by Tet1 to be responsible for the complete reprogramming, which is consistent with the recent studies showing that Tet1 deletion in ESCs and the subsequent reduction of cellular $5 \mathrm{hmC}$ did not affect the expressions of key pluripotent genes including Oct4, Nanog, and Sox2, as well as ESC pluripotency [56-58]. Interestingly, activated Stat 3 also suppressed Tet 1 expression in MEFs (Fig. 4A). Thus, our data suggest an upregulation of Tet1 expression by unidentified factor(s), which is positively regulated by Stat3 signaling in the context of late-reprogramming stage.

Chromatin modification by histone acetylation is closely associated with DNA demethylation, and HDAC inhibitors significantly improve the induction efficiency of iPSCs $[59,60]$. We therefore investigated the effect of Jak/Stat 3 inhibition on the expression of class I HDACs during reprogramming. Analysis by qRT-PCR revealed that HDACs 2, 3, and 8 expressions were mildly but significantly elevated in day-18 OKSM-transduced cells treated with Jaki over those treated with DMSO in the control group (Fig. 4B). This was further confirmed in the isolated colonies described in the previous result section, with at least two- to fourfold increase for their gene expression by Jaki treatment (Fig. 4C). In addition to the increased expression of HDACs, we also observed significant increases in the expression of $\mathrm{AOF} 1, \mathrm{MeCP} 2$, and methyl-CpG binding protein 

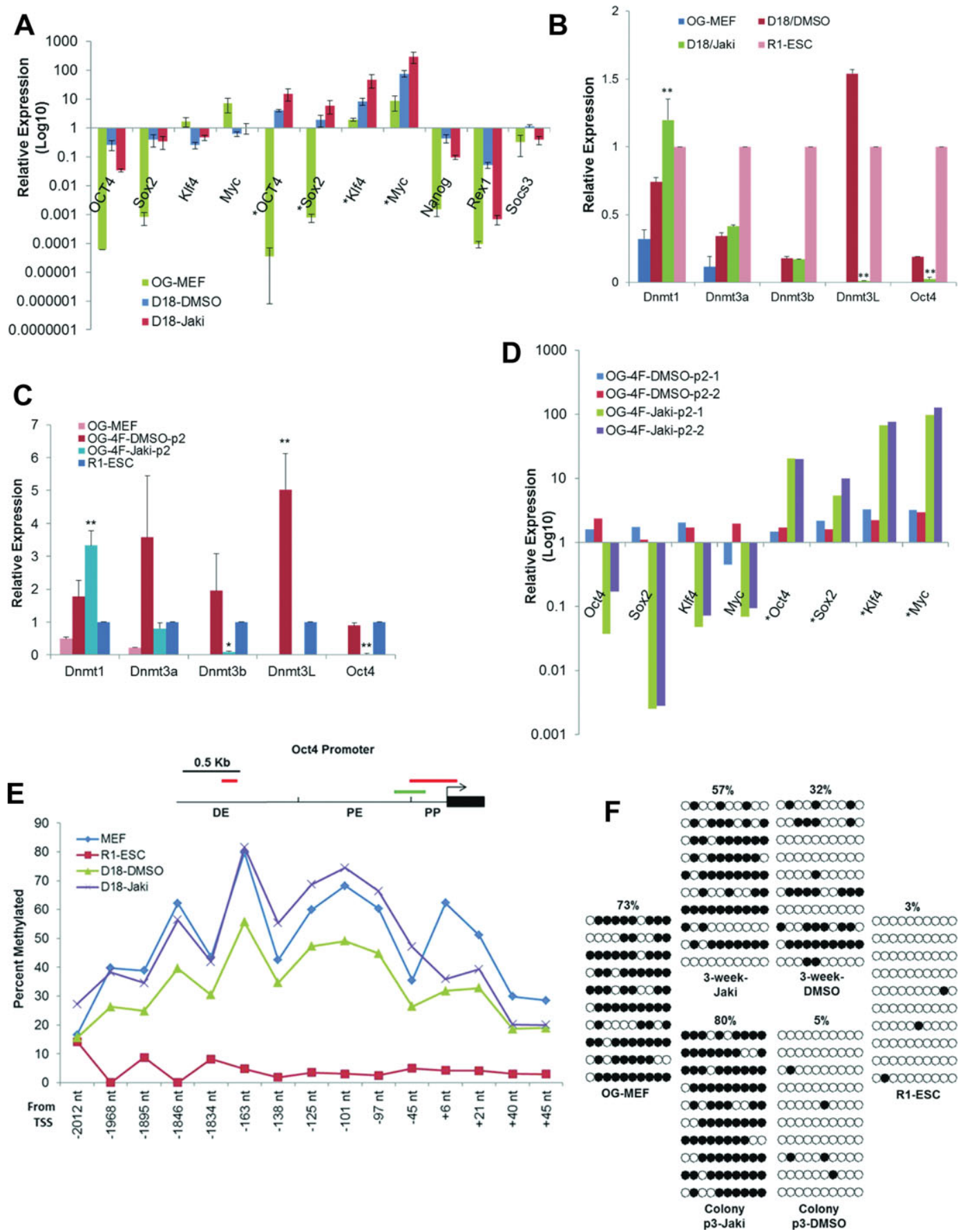

Figure 3. Janus kinase/signal transducer and activator of transcription 3 (Jak/Stat3) activity regulates DNA methylation during reprogramming. (A): Relative levels of mRNA (mean \pm SD) in MEFs, R1-ESCs, and Oct4, Klf4, Sox2, and c-Myc (OKSM)-transduced MEFs 18 days after initial viral transduction. The cells were treated with either DMSO or $1 \mu \mathrm{M} \mathrm{Jaki}\left({ }^{*} \mathrm{Oct} 4\right.$, *Sox $2,{ }^{*} \mathrm{Klf} 4$, *Myc: combined retroviral transgene and endogenous gene expression. Others represent endogenous gene expression only. Values were normalized with glyceraldehyde 3-phosphate dehydrogenase $(\mathrm{GAPDH})$, and $\log 10$ transformed relative to those in R1-ESCs, $n=3$ ). (B): Relative levels of mRNA (mean \pm SD) for different Dnmts in MEFs, R1-ESCs, and OKSM-transduced MEFs treated with Jaki or DMSO 18 days after transduction. Values were normalized with GAPDH and relative to R1-ESCs (**, all $p$ values $<.01, n=3$ ). (C): Relative levels of mRNA (mean $\pm \mathrm{SD}$ ) for different Dnmts in colonies isolated from OKSM-induced MEF 3 weeks after viral transduction. The cells were treated with either Jaki or DMSO and cultured to passage 2 (4F: OKSM). Results were normalized with GAPDH and relative to R1-ESCs (**, all $p$ values $<.01, n=3$ ). (D): Relative expression levels of pluripotent transcription factors in colonies isolated 3 weeks after transduction by OKSM and treated with either Jaki or DMSO treatment. The cells were cultured to passage 2 (4F: OKSM. *Oct4, *Sox 2 , *Klf4, and *Myc: combined retroviral and endogenous gene expression). The others represent endogenous gene expression only. The average values of duplicate sample preparations were normalized by GAPDH, $\log 10$ transformed and relative to R1-ESCs. (E): Upper panel: Schematic diagram of the Oct4 gene at the DE, PE, and PP regions. Arrow denotes transcription start site (TSS). Red and green bars denote regions for pyrosequencing and bisulfite DNA sequencing, respectively. Lower panel: Representative illustrations of DNA pyrosequencing results at the Oct4 DE and PP region in OKSM-transduced MEFs treated with $1 \mu$ M Jaki or DMSO 18 days after OKSM transduction. MEFs and R1-ESCs served as the controls. The numbers under the $x$-axis indicate the distance of each CpG dinucleotide from the TSS. (F): Bisulfite DNA sequencing analysis to part of the Oct4 PE and PP regions in MEFs treated with DMSO or Jaki 3 weeks after OKSM transduction and isolated colonies cultured to passage 3. MEF and R1-ESCs served as the controls. Filled and open circles represent methylated and unmethylated CpGs, respectively. The percentage of total methylated CpGs for each analyzed region was given on top of each dataset. Abbreviations: DMSO, dimethyl sulfoxide; DE, distal enhancer; Dnmt, DNA methyltransferase; ESC, embryonic stem cell; MEF, mouse embryonic fibroblasts; PE, proximal enhancer; PP, proximal promoter. 

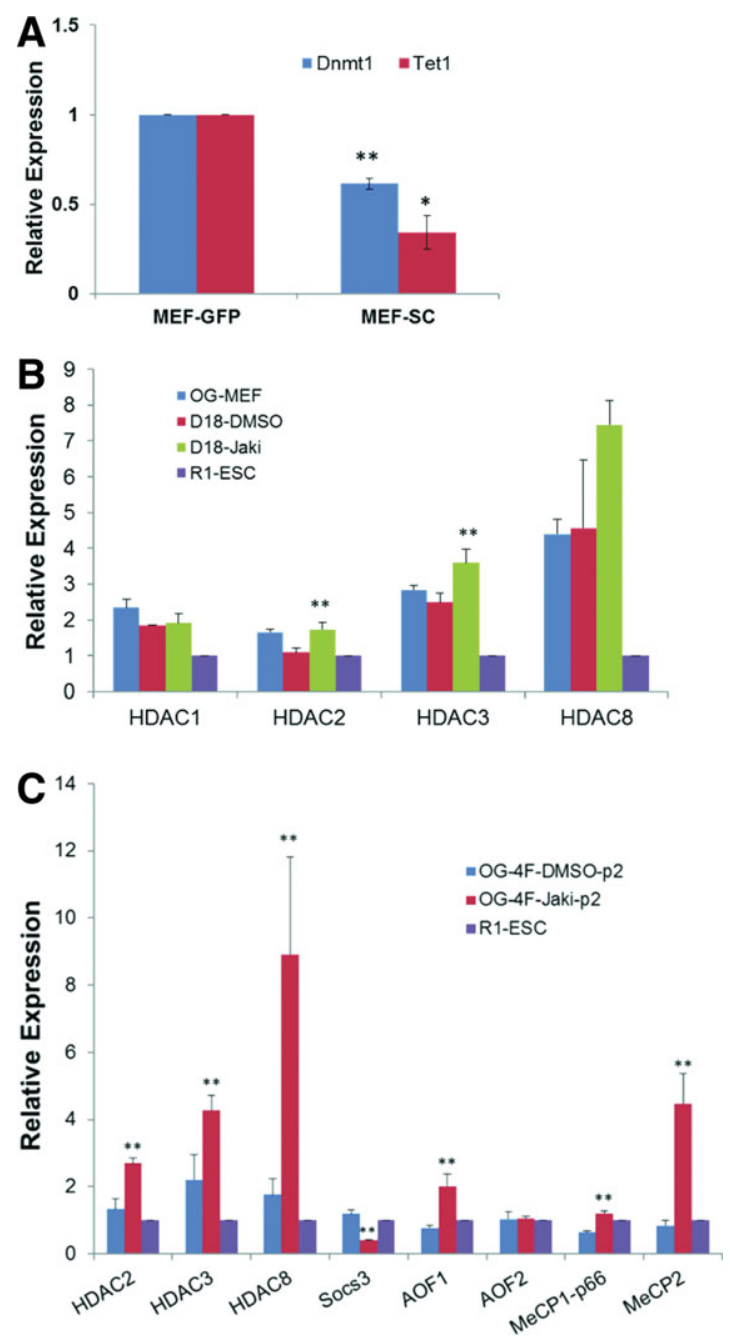

Figure 4. Janus kinase/signal transducer and activator of transcription 3 (Jak/Stat3) activity suppresses Dnmt1 and HDAC expressions during reprogramming. (A): Relative expression levels of mRNA (mean \pm SD) for Dnmt1 and Tet1 in B6/129 MEFs transduced with retroviral GFP or Stat3C as described in Figure 2(B) for 4 days. Values were normalized with glyceraldehyde 3-phosphate dehydrogenase $(\mathrm{GAPDH})$ and relative to GFP control (**, $p=.001 ;{ }^{*}, p=.014, n=$ 2). (B): Relative expression levels of mRNA (mean \pm SD) for different HDACs in MEFs, R1-ESCs, and Oct4, Klf4, Sox2, and c-Myc (OKSM)-transduced MEFs treated with Jaki or DMSO at 18 days after the initial transduction. Values were normalized with GAPDH and relative to R1-ESCs (**, all $p$ values $<.01, n=3$ ). (C): Relative levels of mRNA (mean $\pm \mathrm{SD}$ ) for different HDACs, AOF1/2, and MeCP1-p66/ $\mathrm{MeCP} 2$ in isolated colonies induced by OKSM and treated with Jaki or DMSO at 3 weeks after viral transduction. The cells were analyzed at passage 2 (4F: OKSM). Values were normalized with GAPDH and relative to R1-ESCs (**, all $p$ values $<.01, n=3$ ). Abbreviations: Dnmt, DNA methyltransferase; DMSO, dimethyl sulfoxide; ESC, embryonic stem cell; GFP, green fluorescent protein; HDAC, histone deacetylase; MEF, mouse embryonic fibroblast; SC, Stat3C.

1-p66 (MeCP1-p66, also known as GATAD2B) in Jaki-treated colonies over DMSO controls (Fig. 4C). AOF1 promotes H3K4 demethylation, and MeCP2/1-p66 directly binds to methylated DNAs and recruits HDACs, which are associated with chromatin condensation and gene silencing [61-63]. Downregulation of these mRNA transcripts by ESC-specific microRNA mir-302 was found to play a significant role in global DNA demethylation during reprogramming [64]. Taken together, our findings indicate that Jak/Stat 3 activity suppresses Dnmt1 and HDACs expression, as well as the expression of other repressive chromatin regulators during reprogramming, thus facilitating DNA demethylation and open-chromatin formation at pluripotent loci. These potentiate the complete activation of pluripotency related genes by allowing full access of transcriptional machineries.

\section{Dnmt or HDAC Inhibition but not Nanog Overexpression Significantly Rescues Reprogramming Arrested by Jak/Stat3 Inactivation}

We have found that Jak/Stat3 inhibition resulted in significantly lower Nanog activation than the DMSO control at day 18 of MEF reprogramming by OKSM (Fig. 3A). To test whether the inadequate Nanog expression caused the incomplete Oct4 activation under Jak/Stat3 inhibition, MEFs were transduced with retroviral OKSM plus Nanog (OKSMN) and reprogrammed without feeder cells. Reprogramming without LIF or with LIF plus Jaki both blocked the GFP+ colony formation regardless of Nanog overexpression (Fig. 5A; supporting information Fig. S13). Thus, insufficient Nanog expression does not appear to be the direct cause for the trapped reprogramming, and Nanog overexpression does not rescue the impeded MEF reprogramming resulting from LIF deprivation or Jak/Stat3 inhibition.

To test whether the elevated expression of Dnmt1 or HDACs represents a significant cause for the reprogramming arrested by Jak/Stat 3 inhibition at late stage, we transduced MEFs with OKSM and treated them with LIF plus Jaki starting at day 3 as described earlier. As Dnmt inhibition at early reprogramming stage resulted in cell death [16], we started adding the Dnmt inhibitor, 5-Aza-2'-deoxycytidine (AZA), or the HDAC inhibitor, valproic acid (VPA) and trichostatin A (TSA) to the culture medium at day 9 until day 15 . Treatment with AZA in the presence of Jak/Stat 3 inhibition resulted in a 2.7-fold increase in the number of GFP + colonies by day 15 , while treating the cells with VPA produced a milder (1.7fold) increase in GFP + colonies, compared to the Jaki treatment control (Fig. 5B). Upon removing Jaki and all other inhibitors and allowing the cells (including the control) to continue reprogramming for three additional days in LIF containing medium, we could still observe a significant twofold and 1.7-fold increase in GFP+ colonies from the AZA or VPA treated cells, respectively, compared to the control (Fig. $5 \mathrm{~B})$. Although the combined treatment of AZA/VPA resulted in significant cytotoxicity in our study (data not shown), it is possible that a combined Dnmt/HDACs inhibition with less cytotoxic and more specific action (such as small interfering RNA) may result in a complete rescue of the Jaki-arrested reprogramming. These results indicated that inhibition of Dnmt1 or HDACs at least partially and significantly rescues the reprogramming arrested by Jak/Stat 3 inhibition.

To address further whether elevated HDAC expression is a significant cause for the trapped reprogramming by the loss of Jak/Stat3 activity, we transduced MEFs with OKSM and deprived them of residual LIF with the LIF-antibody starting at day 3 after viral transduction [65]. The cells were then further treated with VPA or TSA starting at day 9 until day 15 of OKSM transduction. The VPA treatment resulted in a significant sixfold increase in the number of GFP+ colonies at day 15 compared to the LIF-antibody only control (Fig. 5C). After replacing LIF antibody and all the inhibitors with LIFcontaining medium (including the LIF-antibody control) for an additional 6 days, the VPA-treated cells still showed a 3.6fold increase in GFP+ colonies over the controls (Fig. 5C). Furthermore, the percentage of GFP + cells in VPA treatment 
A

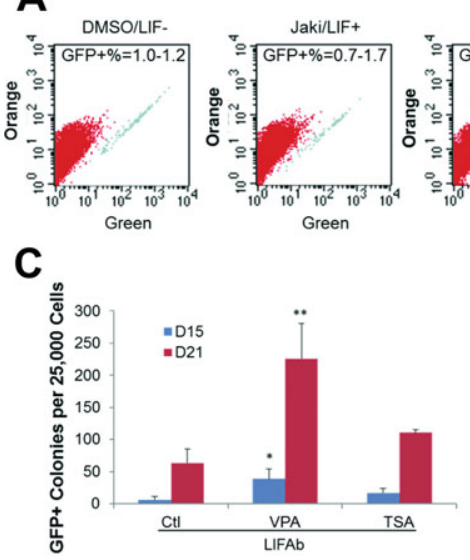

E

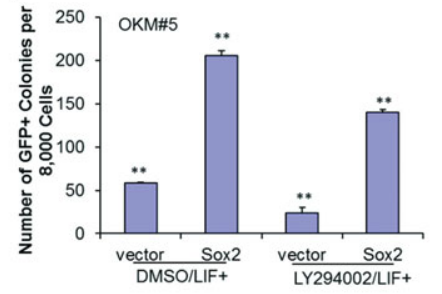

$\mathbf{F}$

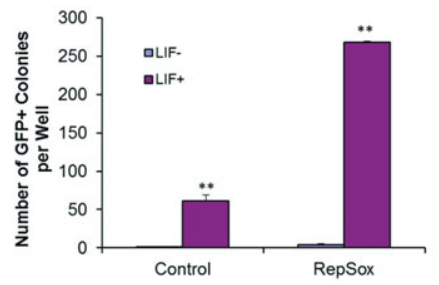

B $\frac{\underline{\bar{c}}}{\mathrm{c}}$

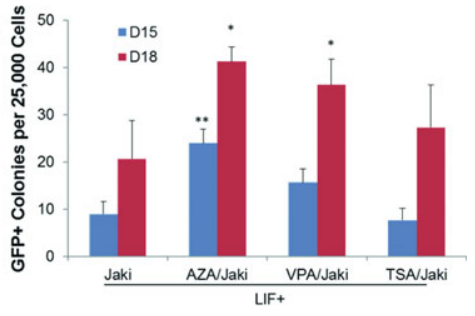

D
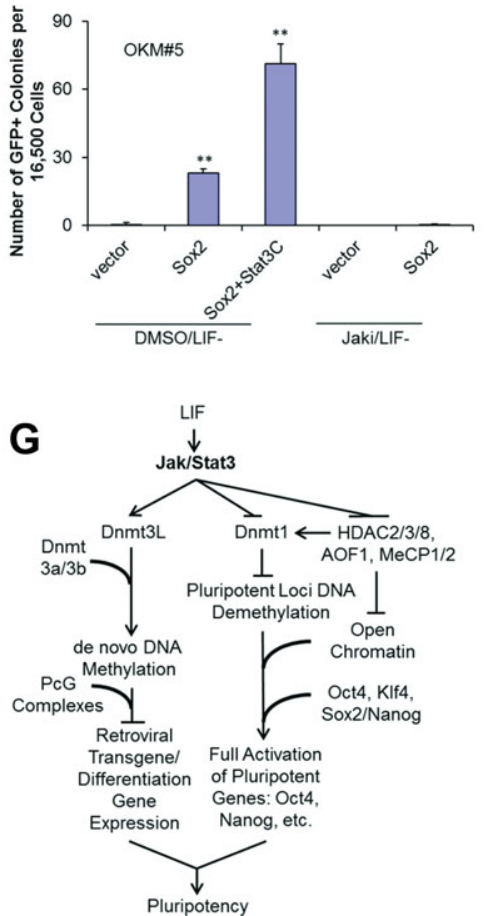

Figure 5. Dnmt or HDAC inhibition but not Nanog expression rescues impeded reprogramming by Jak/Stat3 inhibition, and Jak/Stat3 activity is essential for OKM-pre-iPSC reprogramming. (A): Fluorescence activated cell sorting (FACS) of GFP+ cells 3 weeks after viral OKSM plus Nanog (OKSMN) transduction of MEFs and reprogrammed without feeders. Cells were treated without LIF or with LIF plus DMSO or Jaki (the percentages of GFP + cells, GFP $+\%$, in total live cells were given in the representative figures; $n=2)$. (B): The number of GFP + colonies (mean \pm SD) 15 and 18 days after OKSM transduction of MEFs cultured in LIF containing medium. Cells were treated with Jaki starting on day 3 , and AZA $(0.5 \mu \mathrm{M})$, VPA $(1 \mathrm{mM})$, or TSA $(20 \mathrm{nM})$ was added in medium from day 9 to day 15 . All inhibitors were washed away, and cells were cultured in LIF-containing medium from day $15-18$, results represent means from three individual wells $(* *, p=.000, *, p=.024)$. (C): The number of GFP+ colonies (mean \pm SD) 15 and 21 days after OKSM transduction of mouse embryonic fibroblasts (MEFs) cultured without LIF and feeder cells. LIFAb was added starting at day 3, VPA $(1 \mathrm{mM})$ or TSA $(20 \mathrm{nM})$ was added the same as described in (B). The medium was then switched to LIF-containing medium for days $15-21$, results represent means from three individual wells $(*, p=.022 ; * *, p=.003)$. (D): The number of GFP+ colonies (mean $+\mathrm{SD}$ ) from OKM\#5 pre-iPSCs. The cells were transduced with Sox2, Sox 2 plus Stat3C, or control retroviral vector for 24 hours, and then cultured in knockout serum replacement (KSR)-ESC medium without LIF, and treated with either DMSO or $1 \mu \mathrm{M}$ Jaki. GFP + colonies were scored at 10 days after viral transduction. $(* *, p=.000, n=2)$. $(\mathbf{E})$ : The number of GFP + colonies $($ mean \pm SD) from OKM\#5 pre-iPSCs. The cells were transduced with Sox2, Sox2 plus Stat3C, or control retroviral vector and cultured in KSR-ESC medium with LIF and treated with either DMSO or $10 \mu \mathrm{M}$ LY294002. GFP+ colonies were scored 10 days after viral transduction $(* *, p=$ $.000, n=2)$. $(\mathbf{F})$ : The number of GFP + colonies (mean \pm SD) from OKM\#5 pre-iPSCs. Cells were treated with either DMSO control or $25 \mu \mathrm{M}$ RepSox for 24 hours and then cultured in KSR-medium with or without LIF. GFP+ colonies were scored at 10 days after chemical treatment $(* *, p=.000, n=2)$. (G): The proposed model of Jak/Stat3 in promoting pluripotency establishment in murine somatic cell reprogramming. LIF signal activates Jak/Stat3, which suppresses Dnmt1, HDACs, AOF1, and MeCP1-p66/MeCP2 expressions, and stimulates Dnmt3L/3b expressions. Suppression of Dnmt1/HDACs/AOF1/MeCP1-p66/MeCP2 facilitates the DNA demethylation at the pluripotent loci and the establishment of an open chromatin structure, enabling these genes for complete activation. Dnmt3L expression stimulates the de novo DNA methylation activities of Dnmt3a/3b, which silences retroviral transgenes and probably linage commitment genes, in addition to Polycomb-mediated gene suppression. Together these epigenetic modulations mediated by Jak/Stat3 signaling promote the pluripotency establishment during reprogramming. Abbreviations: AZA, 5-Aza-2'-deoxycytidine; DMSO, dimethyl sulfoxide; Dnmt, DNA methyltransferase; GFP, green fluorescent protein; HDAC, histone deacetylase; Jak/Stat3, Janus kinase/signal transducer and activator of transcription 3; LIF, leukemia inhibitory factor; LIFAb, LIF antibody; OKM, Oct4, Klf4, and c-Myc; TSA, trichostatin A; VPA, valproic acid.

at day 23 was almost identical to the positive control cells treated by LIF (without LIF-antibody), which is 12 -fold greater than the LIF-antibody only control (supporting information Fig. S14).
Interestingly, we noticed that VPA treatment showed a more prominent rescue to the reprogramming arrested by the LIF-antibody than by Jaki treatment. One possible reason could be a difference in Erk activity, which impairs ESC self- 
renewal and promotes differentiation [27, 39]. As LIF stimulation strongly phosphorylates Erk1/2 in MEFs and LIFstarved ESCs/iPSCs as shown in our study (Fig. 1A) and previous research [25, 27], in Jaki treatment the Erk1/2 were strongly activated in the presence of LIF. However, under LIF-antibody conditions the VPA treatment could exert a more significant rescue to the arrested reprogramming, due to attenuated Erk differentiation signal without LIF cytokine, under our reprogramming condition.

Therefore, temporary inhibition of Dnmt or HDAC during the late-reprogramming stage can significantly revert the arrested reprogramming in the presence of either Jak/Stat 3 inhibition or LIF deprivation. Taken together, our results indicated that elevated expressions of Dnmt1 and HDACs but not low levels of Nanog expression significantly contribute to reprogramming arrested by Jak/Stat 3 inhibition.

\section{LIF/Stat3 Signaling Is Essential for Sox2 Overex- pression or TGF $\beta$ Inhibition-Induced Pre-iPSC Reprogramming}

The retroviral OKM-induced pre-iPSCs (OKM-pre-iPSCs) can be completely reprogrammed to pluripotency by overexpressing Sox 2 or Nanog [66]. Because we had determined that Jak/ Stat 3 activation is essential for the late-stage reprogramming of MEFs, we asked whether it is also required for the complete reprogramming of OKM-pre-iPSCs. Nine out of 12 GFP- OKM-pre-iPSC cell lines responded to the retroviral Sox2 induction, but only two responded to the retroviral Nanog induction by expressing Oct4-GFP (supporting information Fig. S15). We chose OKM-pre-iPSC\#5 and \#18 (OKM\#5 or \#18) to study the role of Jak/Stat3 in Sox2-mediated complete reprogramming further. In the absence of LIF, Stat3C expression significantly improved the Sox2-induced complete reprogramming of both OKM\#5 and \#18, whereas Jaki treatment blocked the GFP + colony formation (Fig. 5D; supporting information Fig. S16). Similar to MEF reprogramming, inhibition of PI3K/Akt activity by LY294002 only partially reduced but did not block the Sox2-induced reprogramming of either pre-iPSC cell line (Fig. 5E; supporting information Fig. S17). These results demonstrated that Jak/ Stat 3 activation is required for Sox2-induced complete reprogramming of OKM-pre-iPSCs.

The transforming growth factor beta (TGF $\beta$ ) receptor kinase inhibitor RepSox promotes complete reprogramming of OKM-pre-iPSCs by stimulating endogenous Nanog expression [66]. We asked whether the reprogramming by TGF $\beta$ pathway inhibition also depends on LIF signaling. In the presence of LIF, treatment by RepSox greatly stimulated the reprogramming of OKM\#5 pre-iPSCs (Fig. 5F). However, the reprogramming effect was completely diminished in the absence of LIF (Fig. 5F; supporting information Fig. S18). Thus, the complete reprogramming of OKM-pre-iPSCs promoted by inhibiting the TGF- $\beta$ receptor also depends on LIF signaling.

\section{Discussion}

We demonstrated here that elevated Stat 3 activity significantly enhances the pluripotency establishment of MEFs induced by either OKS or OKSM. Enhanced Stat3 activation also improves the total colony formation in OKS reprogramming. The gene c-Myc is a direct target of Stat 3 in ESCs. Constitutively activated c-Myc expression in ESCs maintains ESC pluripotency in the absence of LIF [32, 67]. Addition of c-Myc improves OKS reprogramming by promoting cell proliferation thus accelerating the reprogramming process [68].
This could explain why the enhanced Stat3 activation increased the total colony formation in OKS but not in OKSM transduction. Although we did not detect an upregulation of c-Myc in MEFs transfected with Stat3C (data not shown), it is possible that the enhanced Stat3 activation stimulated c-Myc expression in partially dedifferentiated cells during the reprogramming, which further promoted cell proliferation and colony formation. However, in OKSM reprogramming, this effect of Stat 3 would be rendered insignificant by the overexpression of retroviral c-Myc.

Here, we demonstrated the essential role of Jak/Stat3 in acquiring pluripotency during late-stage MEF reprogramming. The constitutive retroviral transgene expression in the absence of Jak/Stat3 activity may partially contribute to the incomplete reprogramming. Silencing of retroviral transgenes is a prerequisite for pluripotency establishment in retroviral mediated reprogramming, as overexpression of pluripotent genes, especially Oct4, leads to differentiation of ESCs [13, 17, 18]. It was demonstrated that Dnmt3L is the essential component for retroviral silencing in ESCs by stimulating Dnmt3a/3b activities, and retroviral expressing/silencing has been the tool to study de novo methylation in pluripotent cells $[19,23]$. Dnmt3a, 3b, and 3L are mainly expressed in ESCs and embryonic stages and are responsible for the proper establishment of imprinting during gametogenesis [69-72]. Mice lacking Dnmt3L exhibit imprinting defect [71, 72].

We discovered that Jak/Stat3 inhibition completely blocks Dnmt3L expression and thus abrogates the stimulation of de novo methylation activity specific for retroviral silencing during reprogramming. In addition to de novo methylation, Dnmt3L is also required to maintain the DNA methylation patterns in ESCs [23]. Our finding that Jak/Stat3 activity stimulates Dnmt3L expression therefore sheds new light on the role played by LIF signaling for both pluripotency attainment and maintenance.

We demonstrated that inhibition of Jak/Stat3 blocks DNA demethylation of Oct4 and Nanog regulatory regions during reprogramming, which is marked by significantly suppressed endogenous pluripotent gene expression. Extensive epigenetic change happens during the reprogramming process [16, 7375]. The promoter regions of pluripotent genes including Oct4, Nanog, and Rex1 are hypermethylated in MEFs and pre-iPSCs $[2,16]$ and hypomethylated in iPSCs and ESCs $[1$, $3,13,76,77]$. The efficiency for complete reprogramming of somatic cells and pre-iPSCs by OKSM can be improved several-fold by inhibiting Dnmt1 with AZA or Dnmt1 specific short hairpin RNAs $[16,59,78]$. In mouse placenta and trophoblast stem cells, where the Oct4 promoter region is hypermethylated, depletion or inhibition of Dnmt1 resulted in hypomethylation of the Oct4 promoter region and ectopic Oct4 expression [15]. These previous studies had established a strong connection between Oct4 gene activation and DNA demethylation promoted by suppression of Dnmt1 activity in the context of specific developmental stages.

Histone acetylation is closely associated with DNA demethylation in the Oct4 promoter region in ESCs [2, 15], and the HDAC inhibitor sodium butyrate was shown to improve Oct4 promoter demethylation during OKSM reprogramming of fibroblasts [60]. Inhibition of HDAC activity promotes the ubiquitin-dependent proteasomal degradation of Dnmt1 [79, 80], further suggesting a synergistic promotion of DNA demethylation by suppression of both Dnmt1 and HDAC activities. Although a passive model of genomic DNA demethylation as a result of inhibited methylation maintenance has been proposed for reprogramming $[81,82]$, the actual mechanism that the cells may control this process under extracellular cues has remained unclear. 
We found that during reprogramming, the hypermethylation of Oct4 and Nanog enhancer/promoter regions as the result of Jak/Stat3 inhibition is tightly correlated with the significantly elevated Dnmt1 and HDAC 2, 3, 8 expressions, compared to the control reprogrammed cells. Accordingly, we demonstrated that inhibiting Dnmt or HDAC activity by AZA or VPA partially but significantly rescues the reprogramming arrested by Jak/Stat3 inhibition or LIF deprivation. Our data thus strongly argue that Jak/Stat3 signaling plays a critical function in facilitating the demethylation of endogenous pluripotent genes and in promoting the formation of open-chromatin structure by suppressing the expression of Dnmt1 and HDACs during reprogramming. In addition, we also found that loss of Jak/Stat3 activity leads to the significant increase of $\mathrm{AOF} 1, \mathrm{MeCP} 1-\mathrm{p} 66$, and $\mathrm{MeCP} 2$ expression. Downregulation of AOF1/2 and MeCP1-p66/MeCP2 mRNAs and the subsequent destabilization and degradation of Dnmt1 protein have been indicated to be the mechanism of mir-302 expression mediated global DNA demethylation, which triggers the activation of pluripotent genes [64, 83, 84]. Together, our findings supported the central role of Jak/Stat 3 activity in the key epigenetic regulation events during reprogramming, which prepares and potentiates the pluripotent genes for complete activation by transcriptional machineries.

Repressive-chromatin represents a major mechanical barrier to transcription factor induced reprogramming [85]. Oct4, Sox2, and Nanog function as the core pluripotent gene circuitry in ESCs [86, 87]. The Oct4 gene enhancer/promoter region contains binding sites for Oct 4 , Sox 2 , and Nanog proteins to activate its own transcription $[87,88]$.

We found that overexpression of Nanog together with the transcription factor OKSM does not overcome the LIF deprivation or Jak/Stat3 inhibition for complete MEF reprogramming, which is marked by activation of endogenous Oct4 expression. In addition, both Sox 2 expression and TGF $\beta$ signal inhibition failed to induce pluripotency in OKM-preiPSCs without Jak/Stat3 activation or LIF signaling. Therefore, the Jak/Stat 3 activation represents a prerequisite for pluripotency establishment during the reprogramming of both terminally differentiated and partially reprogrammed cells.

The evidence we presented here thus support a model (Fig. 5G) where Jak/Stat3 activation orchestrates the major epigenetic events during reprogramming, to establish the open-chromatin structure in pluripotent loci for the full access of transcription factor complexes, thus allowing complete

\section{REFERENCES}

1 Okita K, Ichisaka T, Yamanaka S. Generation of germline-competent induced pluripotent stem cells. Nature 2007;448:313-U311.

2 Takahashi K, Yamanaka S. Induction of pluripotent stem cells from mouse embryonic and adult fibroblast cultures by defined factors. Cell 2006;126:663-676.

3 Yu J, Vodyanik MA, Smuga-Otto K et al. Induced pluripotent stem cell lines derived from human somatic cells. Science 2007;318: 1917-1920.

4 Anokye-Danso F, Trivedi CM, Juhr D et al. Highly efficient miRNAmediated reprogramming of mouse and human somatic cells to pluripotency. Cell Stem Cell 2011;8:376-388.

5 Kim D, Kim CH, Moon JI et al. Generation of human induced pluripotent stem cells by direct delivery of reprogramming proteins. Cell Stem Cell 2009;4:472-476.

6 Miyoshi N, Ishii H, Nagano $\mathrm{H}$ et al. Reprogramming of mouse and human cells to pluripotency using mature microRNAs. Cell Stem Cell 2011;8:633-638.

7 Warren L, Manos PD, Ahfeldt T et al. Highly efficient reprogramming to pluripotency and directed differentiation of human cells with synthetic modified mRNA. Cell Stem Cell 2010;7:618-630. activation of these genes. On the other hand, Jak/Stat 3 activity stimulates de novo DNA methylation activity which silences retroviral transgenes, and probably linage commitment genes, in addition to Polycomb-group-mediated gene silencing [89]. To the best of our knowledge, this is the first report illustrating the essentialness and the crucial epigenetic role of Jak/ Stat3 activity in the acquisition of pluripotency during latestage reprogramming of murine somatic cells.

\section{Conclusion}

We demonstrated that enhanced Stat3 activation promotes reprogramming of terminally differentiated somatic cells, and Jak/Stat3 activity is essential for complete reprogramming of both terminally differentiated and partially reprogrammed cells. We demonstrated that inhibition of Jak/Stat3 blocks DNA demethylation of Oct 4 and Nanog regulatory regions during reprogramming, which is correlated with elevated expressions of Dnmt 1, HDACs, AOF1, and MeCP2. Inhibiting Jak/Stat3 also blocks Dnmt3L expression, which is correlated with the failure of retroviral silencing. Furthermore, Dnmt or HDAC inhibitor but not Nanog overexpression rescues complete reprogramming in the presence of Jak/Stat 3 inhibition. We conclude that Jak/Stat3 signaling plays a fundamental role in epigenetic regulation to promote pluripotency during late-stage reprogramming.

\section{ACKNOWLEDGMENTS}

We thank Drs. David Goldhamer, Carolyn Teschke, Lawrence Silbart, and Thomas Hoagland for their critical input to our project design, and Dr. Toshio Kitamura of the University of Tokyo for providing PlatE cells. This project was supported by USDA grant (1265-31000-091-02S).

\section{Disclosure of Potential CONFLICTS OF INTEREST}

The authors indicate no potential conflicts of interest.
8 Zhou H, Wu S, Joo JY et al. Generation of induced pluripotent stem cells using recombinant proteins. Cell Stem Cell 2009;4:381-384.

9 Blomen VA, Boonstra J. Stable transmission of reversible modifications: Maintenance of epigenetic information through the cell cycle. Cell Mol Life Sci 2011;68:27-44.

10 De Felici M. Nuclear reprogramming in mouse primordial germ cells: Epigenetic contribution. Stem Cells Int 2011;2011:425863.

11 Hochedlinger K, Plath K. Epigenetic reprogramming and induced pluripotency. Development 2009;136:509-523.

12 Meissner A, Wernig M, Jaenisch R. Direct reprogramming of genetically unmodified fibroblasts into pluripotent stem cells. Nat Biotechnol 2007;25:1177-1181.

13 Wernig M, Meissner A, Foreman R et al. In vitro reprogramming of fibroblasts into a pluripotent ES-cell-like state. Nature 2007;448: 318-324.

14 Nichols J, Zevnik B, Anastassiadis K et al. Formation of pluripotent stem cells in the mammalian embryo depends on the POU transcription factor Oct4. Cell 1998;95:379-391.

15 Hattori N, Nishino K, Ko YG et al. Epigenetic control of mouse Oct4 gene expression in embryonic stem cells and trophoblast stem cells. J Biol Chem 2004;279:17063-17069.

16 Mikkelsen TS, Hanna J, Zhang X et al. Dissecting direct reprogramming through integrative genomic analysis. Nature 2008;454:49-55. 
17 Niwa H, Miyazaki J, Smith AG. Quantitative expression of Oct-3/4 defines differentiation, dedifferentiation or self-renewal of ES cells. Nat Genet 2000;24:372-376.

18 Theunissen TW, van Oosten AL, Castelo-Branco G et al. Nanog overcomes reprogramming barriers and induces pluripotency in minimal conditions. Curr Biol 2011;21:65-71.

19 Okano M, Bell DW, Haber DA et al. DNA methyltransferases Dnmt3a and Dnmt3b are essential for de novo methylation and mammalian development. Cell 1999;99:247-257.

20 Chedin F, Lieber MR, Hsieh CL. The DNA methyltransferase-like protein DNMT3L stimulates de novo methylation by Dnmt3a. Proc Natl Acad Sci USA 2002;99:16916-16921.

21 Suetake I, Shinozaki F, Miyagawa J et al. DNMT3L stimulates the DNA methylation activity of Dnmt3a and Dnmt3b through a direct interaction. J Biol Chem 2004;279:27816-27823.

22 Gowher H, Liebert K. Hermann A et al. Mechanism of stimulation of catalytic activity of Dnmt3A and Dnmt3B DNA-(cytosine-C5)-methyltransferases by Dnmt3L. J Biol Chem 2005;280:13341-13348.

23 Ooi SK, Wolf D, Hartung $\mathrm{O}$ et al. Dynamic instability of genomic methylation patterns in pluripotent stem cells. Epigenetics Chromatin 2010;3:17.

24 Niwa H, Burdon T, Chambers I et al. Self-renewal of pluripotent embryonic stem cells is mediated via activation of STAT3. Genes Dev 1998; 12:2048-2060.

25 Niwa H, Ogawa K, Shimosato D et al. A parallel circuit of LIF signalling pathways maintains pluripotency of mouse ES cells. Nature 2009;460:118-122.

26 Paling NR, Wheadon $\mathrm{H}$, Bone $\mathrm{HK}$ et al. Regulation of embryonic stem cell self-renewal by phosphoinositide 3-kinase-dependent signaling. J Biol Chem 2004;279:48063-48070.

27 Burdon T, Smith A, Savatier P. Signalling, cell cycle and pluripotency in embryonic stem cells. Trends Cell Biol 2002;12:432-438.

28 Heinrich PC, Behrmann I, Muller-Newen G et al. Interleukin-6-type cytokine signalling through the gp130/Jak/STAT pathway. Biochem J 1998;334(Pt 2):297-314.

29 Taga T, Kishimoto T. Gp130 and the interleukin-6 family of cytokines. Annu Rev Immunol 1997;15:797-819.

30 Matsuda T, Nakamura T, Nakao K et al. STAT3 activation is sufficient to maintain an undifferentiated state of mouse embryonic stem cells. EMBO J 1999;18:4261-4269.

31 Hall J, Guo G, Wray J et al. Oct4 and LIF/Stat3 additively induce Kruppel factors to sustain embryonic stem cell self-renewal. Cell Stem Cell 2009:5:597-609.

32 Cartwright P, McLean C, Sheppard A et al. LIF/STAT3 controls ES cell self-renewal and pluripotency by a Myc-dependent mechanism. Development 2005;132:885-896.

33 Ying QL, Wray J, Nichols $\mathrm{J}$ et al. The ground state of embryonic stem cell self-renewal. Nature 2008;453:519-523.

34 van Oosten AL, Costa Y, Smith A et al. JAK/STAT3 signalling is sufficient and dominant over antagonistic cues for the establishment of naive pluripotency. Nat Commun 2012;3:817.

35 Yang J, van Oosten AL, Theunissen TW et al. Stat3 activation is limiting for reprogramming to ground state pluripotency. Cell Stem Cell 2010;7:319-328.

36 Bromberg JF, Wrzeszczynska MH, Devgan G et al. Stat3 as an oncogene. Cell 1999;98:295-303.

37 Burdon T, Chambers I, Stracey $\mathrm{C}$ et al. Signaling mechanisms regulating self-renewal and differentiation of pluripotent embryonic stem cells. Cells Tissues Organs 1999;165:131-143.

38 Schmitz J, Weissenbach M, Haan S et al. SOCS3 exerts its inhibitory function on interleukin-6 signal transduction through the SHP2 recruitment site of gp130. J Biol Chem 2000;275:12848-12856.

39 Burdon T, Stracey C, Chambers I et al. Suppression of SHP-2 and ERK signalling promotes self-renewal of mouse embryonic stem cells. Dev Biol 1999;210:30-43.

40 Ernst M, Najdovska M, Grail D et al. STAT3 and STAT1 mediate IL11-dependent and inflammation-associated gastric tumorigenesis in gp130 receptor mutant mice. J Clin Invest 2008;118:1727-1738.

41 Silva J, Nichols J, Theunissen TW et al. Nanog is the gateway to the pluripotent ground state. Cell 2009;138:722-737.

42 Kim J, Efe JA, Zhu S et al. Direct reprogramming of mouse fibroblasts to neural progenitors. Proc Natl Acad Sci USA 2011;108: 7838-7843.

43 Rathjen PD, Toth S, Willis A et al. Differentiation inhibiting activity is produced in matrix-associated and diffusible forms that are generated by alternate promoter usage. Cell 1990;62:1105-1114.

44 Stewart CL, Kaspar P, Brunet LJ et al. Blastocyst implantation depends on maternal expression of leukaemia inhibitory factor. Nature 1992;359:76-79.

45 Dong KB, Maksakova IA, Mohn F et al. DNA methylation in ES cells requires the lysine methyltransferase G9a but not its catalytic activity. EMBO J 2008;27:2691-2701.
46 Leung DC, Dong KB, Maksakova IA et al. Lysine methyltransferase G9a is required for de novo DNA methylation and the establishment, but not the maintenance, of proviral silencing. Proc Natl Acad Sci USA 2011;108:5718-5723.

47 Hermann A, Goyal R, Jeltsch A. The Dnmt1 DNA-(cytosine-C5)methyltransferase methylates DNA processively with high preference for hemimethylated target sites. J Biol Chem 2004;279:48350-48359.

48 Szyf M. The role of DNA hypermethylation and demethylation in cancer and cancer therapy. Curr Oncol 2008;15:72-75.

49 Torrisani J, Unterberger A, Tendulkar SR et al. AUF1 cell cycle variations define genomic DNA methylation by regulation of DNMT1 mRNA stability. Mol Cell Biol 2007;27:395-410.

50 Dhe-Paganon S, Syeda F, Park L. DNA methyl transferase 1: Regulatory mechanisms and implications in health and disease. Int $\mathrm{J}$ Biochem Mol Biol 2011;2:58-66.

51 Snyder M, Huang XY, Zhang JJ. Identification of novel direct Stat3 target genes for control of growth and differentiation. J Biol Chem 2008;283:3791-3798.

52 Guo JU, Su Y, Zhong C et al. Hydroxylation of 5-methylcytosine by TET1 promotes active DNA demethylation in the adult brain. Cell 2011;145:423-434

53 Tahiliani M, Koh KP, Shen Y et al. Conversion of 5-methylcytosine to 5-hydroxymethylcytosine in mammalian DNA by MLL partner TET1. Science 2009;324:930-935.

54 Williams K, Christensen J, Pedersen MT et al. TET1 and hydroxymethylcytosine in transcription and DNA methylation fidelity. Nature 2011;473:343-348.

$55 \mathrm{Wu} \mathrm{H}$, D'Alessio AC, Ito S et al. Dual functions of Tet1 in transcriptional regulation in mouse embryonic stem cells. Nature 2011;473: 389-393.

56 Koh KP, Yabuuchi A, Rao S et al. Tet1 and Tet2 regulate 5-hydroxymethylcytosine production and cell lineage specification in mouse embryonic stem cells. Cell Stem Cell 2011;8:200-213.

57 Dawlaty MM, Ganz K, Powell BE et al. Tet1 is dispensable for maintaining pluripotency and its loss is compatible with embryonic and postnatal development. Cell Stem Cell 2011;9:166-175.

58 Ficz G, Branco MR, Seisenberger S et al. Dynamic regulation of 5hydroxymethylcytosine in mouse ES cells and during differentiation. Nature 2011;473:398-402.

59 Huangfu D, Maehr R, Guo W et al. Induction of pluripotent stem cells by defined factors is greatly improved by small-molecule compounds. Nat Biotechnol 2008;26:795-797.

60 Mali P, Chou BK, Yen J et al. Butyrate greatly enhances derivation of human induced pluripotent stem cells by promoting epigenetic remodeling and the expression of pluripotency-associated genes. Stem Cells 2010;28:713-720.

61 Nan $\mathrm{X}, \mathrm{Ng} \mathrm{HH}$, Johnson $\mathrm{CA}$ et al. Transcriptional repression by the methyl-CpG-binding protein MeCP2 involves a histone deacetylase complex. Nature 1998;393:386-389.

62 Yang Z, Jiang J, Stewart DM et al. AOF1 is a histone H3K4 demethylase possessing demethylase activity-independent repression function. Cell Res 2010;20:276-287.

63 Feng Q, Zhang Y. The MeCP1 complex represses transcription through preferential binding, remodeling, and deacetylating methylated nucleosomes. Genes Dev 2001;15:827-832.

64 Lin SL. Concise review: Deciphering the mechanism behind induced pluripotent stem cell generation. Stem Cells 2011;29:1645-1649.

65 Chou YF, Chen HH, Eijpe $M$ et al. The growth factor environment defines distinct pluripotent ground states in novel blastocyst-derived stem cells. Cell 2008;135:449-461.

66 Ichida JK, Blanchard J, Lam K et al. A small-molecule inhibitor of $\operatorname{TGF} \beta$ signaling replaces sox 2 in reprogramming by inducing nanog. Cell Stem Cell 2009;5:491-503.

67 Varlakhanova NV, Cotterman RF, deVries WN et al. myc maintains embryonic stem cell pluripotency and self-renewal. Differentiation 2010;80:9-19.

68 Wernig M, Meissner A, Cassady JP et al. c-Myc is dispensable for direct reprogramming of mouse fibroblasts. Cell Stem Cell 2008;2: $10-12$.

69 Okano M, Xie S, Li E. Cloning and characterization of a family of novel mammalian DNA (cytosine-5) methyltransferases. Nat Genet 1998; 19:219-220.

70 Bourc'his D, Bestor TH. Meiotic catastrophe and retrotransposon reactivation in male germ cells lacking Dnmt3L. Nature 2004:431:96-99.

71 Bourc'his D, Xu GL, Lin CS et al. Dnmt3L and the establishment of maternal genomic imprints. Science 2001;294:2536-2539.

72 Hata K, Okano M, Lei H et al. Dnmt3L cooperates with the Dnmt3 family of de novo DNA methyltransferases to establish maternal imprints in mice. Development 2002;129:1983-1993.

73 Maherali N, Sridharan R, Xie W et al. Directly reprogrammed fibroblasts show global epigenetic remodeling and widespread tissue contribution. Cell Stem CELL 2007;1:55-70. 
74 Imamura M, Miura K, Iwabuchi K et al. Transcriptional repression and DNA hypermethylation of a small set of ES cell marker genes in male germline stem cells. BMC Dev Biol 2006;6:34.

75 Mikkelsen TS, Ku M, Jaffe DB et al. Genome-wide maps of chromatin state in pluripotent and lineage-committed cells. Nature 2007;448: 553-560.

76 Park IH, Zhao R, West JA et al. Reprogramming of human somatic cells to pluripotency with defined factors. Nature 2008;451:141-146.

77 Takahashi K, Tanabe K, Ohnuki $M$ et al. Induction of pluripotent stem cells from adult human fibroblasts by defined factors. Cell 2007; 131:861-872.

78 Shi Y, Do JT, Desponts C et al. A combined chemical and genetic approach for the generation of induced pluripotent stem cells. Cell Stem Cell 2008;2:525-528.

79 Du Z, Song J, Wang Y et al. DNMT1 stability is regulated by proteins coordinating deubiquitination and acetylation-driven ubiquitination. Sci Signal 2010;3:ra80.

80 Zhou Q, Agoston AT, Atadja P et al. Inhibition of histone deacetylases promotes ubiquitin-dependent proteasomal degradation of DNA methyltransferase 1 in human breast cancer cells. Mol Cancer Res 2008;6:873-883.

81 Hanna J, Saha K, Pando B et al. Direct cell reprogramming is a stochastic process amenable to acceleration. Nature 2009;462:595-601.
82 Hanna JH, Saha K, Jaenisch R. Pluripotency and cellular reprogramming: Facts, hypotheses, unresolved issues. Cell 2010;143:508-525.

83 Lin SL, Chang DC, Chang-Lin S et al. Mir-302 reprograms human skin cancer cells into a pluripotent ES-cell-like state. Rna 2008;14: 2115-2124.

84 Lin SL, Chang DC, Lin CH et al. Regulation of somatic cell reprogramming through inducible mir-302 expression. Nucleic Acids Res 2010;39:1054-1065.

85 Plath K, Lowry WE. Progress in understanding reprogramming to the induced pluripotent state. Nat Rev Genet 2011;12:253-265.

86 Boyer LA, Lee TI, Cole MF et al. Core transcriptional regulatory circuitry in human embryonic stem cells. Cell 2005;122:947-956.

87 Loh YH, Wu Q, Chew JL et al. The Oct4 and Nanog transcription network regulates pluripotency in mouse embryonic stem cells. Nat Genet 2006;38:431-440.

88 Chew JL, Loh YH, Zhang W et al. Reciprocal transcriptional regulation of Pou5f1 and Sox2 via the Oct4/Sox2 complex in embryonic stem cells. Mol Cell Biol 2005;25:6031-6046.

89 Boyer LA, Plath K, Zeitlinger J et al. Polycomb complexes repress developmental regulators in murine embryonic stem cells. Nature 2006;441:349-353.

See www.StemCells.com for supporting information available online. 